\title{
CONVERGENCE PROPERTIES OF A CLASS OF PRODUCT FORMULAS FOR WEAKLY SINGULAR INTEGRAL EQUATIONS
}

\author{
GIULIANA CRISCUOLO, GIUSEPPE MASTROIANNI, AND GIOVANNI MONEGATO
}

ABSTRACT. We examine the convergence of product quadrature formulas of interpolatory type, based on the zeros of certain generalized Jacobi polynomials, for the discretization of integrals of the type

$$
\int_{-1}^{1} K(x, y) f(x) d x, \quad-1 \leq y \leq 1,
$$

where the kernel $K(x, y)$ is weakly singular and the function $f(x)$ has singularities only at the endpoints \pm 1 . In particular, when $K(x, y)=\log |x-y|$, $K(x, y)=|x-y|^{\nu}, \nu>-1$, and $f(x)$ has algebraic singularities of the form $(1 \pm x)^{\sigma}, \sigma>-1$, we prove that the uniform rate of convergence of the rules is $O\left(m^{-2-2 \sigma} \log ^{2} m\right)$ in the case of the first kernel, and $O\left(m^{-2-2 \sigma-2 \nu} \log m\right)$ if $\nu \leq 0$, or $O\left(m^{-2-2 \sigma} \log m\right)$ if $\nu>0$, for the second, where $m$ is the number of points in the quadrature rule.

\section{INTRODUCTION}

The numerical solution of linear Fredholm integral equations of the second kind,

$$
u(y)=\int_{0}^{b} K(|x-y|) u(x) d x+h(y), \quad 0 \leq y \leq b<\infty,
$$

with weakly singular kernels $K(|x-y|)$ containing terms like $\log |x-y|$ or $|x-y|^{\nu}, \nu>-1$, has attracted the attention of several authors; see for instance $[2,10,18,22]$. For this type of equation, the following theoretical result on the degree of smoothness of the unknown $u(x)$ has been proved in [21]; see also $[7,15,17,18]$.

Theorem. In (1.1), assume $h \in C^{n}[0, b], K \in C^{n-1}(0, b]$, for some $n \geq 1$, and

$$
\begin{aligned}
& \left|K^{(i)}(t)\right| \leq \gamma_{i} t^{-p-i} \quad \text { for } 0<t \leq b \text { and } i=0,1, \ldots, n-1, \\
& \left|K^{(i)}(t)\right| \geq \gamma_{i}^{0} t^{-p_{0}-i} \quad \text { for } 0<t \leq t_{0} \text { and } i=0,1, \ldots, n-1,
\end{aligned}
$$

Received February 17, 1989; revised May 30, 1989.

1980 Mathematics Subject Classification (1985 Revision). Primary 65D32.

This work was performed under the auspices of the Italian Ministry of Education (MPI). 
where $p$ and $p_{0}$ are real constants such that $0<p<1$ and $p-(1-p)<p_{0} \leq$ $p$, and $\gamma_{i}, \gamma_{i}^{0}, t_{0}$ are some positive constants. Then, when the homogeneous equation corresponding to (1.1) has only the trivial solution in $C[0, b]$, we have

$$
u \in C[0, b] \cap C^{n}(0, b]
$$

and furthermore,

$\left|u^{(k)}(x)\right| \leq \operatorname{const}\left[x^{-p-k+1}+(b-x)^{-p-k+1}\right], \quad 0<x<b, k=0,1, \ldots, n$.

As a consequence of this theorem, if we want, for example, to solve (1.1) using a numerical procedure of Nyström type, ${ }^{1}$ then we have to be able to deal efficiently with integrals of the form

$$
\int_{-1}^{1} K(|x-y|) f(x) d x
$$

where $f(x)$ contains only endpoint singularities of the type $(1 \pm x)^{\sigma}$ or $(1 \pm x)^{\sigma} \log (1 \pm x)$, since it is well known (see [19]) that the rate of convergence of the numerical method coincides with that of the basic quadrature rule we choose to discretize (1.2).

One possible approach to (1.2) is to use the so-called composite product integration formulas on graded meshes (see [18]); for these rules, optimal convergence results have been given in [18].

In [10], the third author has used whole interval product integration rules of interpolatory type, based on the zeros of Legendre polynomials, to construct a numerical scheme for the linear transport equation; furthermore, in [11] he has presented a general procedure for constructing interpolatory product rules based on any set of distinct knots. For quadratures of this type, the only known general convergence results refer to functions $f(x)$ in $(1.2)$ of class $C^{n}[-1,1]$ (see also [13]); however, these results appear, as confirmed by the case examined in [10], to be very pessimistic when we apply them to functions $f(x)$ which are smooth everywhere in $(-1,1)$ except at the endpoints, where they have mild singularities.

In this paper we consider product rules

$$
\int_{-1}^{1} K(x, y) f(x) d x \approx \sum_{i=1}^{m} w_{m, i}(y) f\left(x_{m, i}\right)
$$

with $K(x, y)=|x-y|^{\nu}, \nu>-1$, or $K(x, y)=\log |x-y|$, of interpolatory type, based on the zeros of some classes of generalized Jacobi orthogonal polynomials. For these we derive convergence estimates which are essential if, for example, we want to know the rate of convergence of the corresponding Nyström type method for (1.1). Further, we extend these estimates to product rules which include among their nodes also the endpoints \pm 1 . Our results of $\S 3$ generalize some of those presented in $[3,4,8,9]$, and can be used, for

\footnotetext{
${ }^{1}$ This procedure is often called product integration; see [18].
} 
example, to obtain convergence estimates for quadrature rules of type (1.3) applied to functions $f(x)$ admitting asymptotic expansions with singular terms like $(1 \pm x)^{\sigma}$ plus a sufficiently smooth remainder.

\section{CONSTRUCTION OF SOME WEIGHTED $L_{1}$-APPROXIMATION POLYNOMIALS} FOR THE FUNCTION $(1-x)^{\sigma}$

Throughout this paper, the symbol "const" stands for a positive constant taking on different values on different occurrences.

The following lemmas are necessary to prove our main theorems.

Lemma 1. The integral

$$
F_{1}(y)=\int_{a}^{1}\left(\sqrt{1-x}+m^{-1}\right)^{\lambda}|x-y|^{\nu} d x,
$$

with $-1 \leq a<1, \lambda<0, \nu>-1$ and $|y| \leq 1$, admits the following bounds:

$$
F_{1}(y) \leq \text { const } \begin{cases}1 & \text { if } \lambda / 2+\nu+1>0, \\ \log m & \text { if } \lambda / 2+\nu+1=0, \\ m^{-2-\lambda-2 \nu} & \text { if } \lambda / 2+\nu+1<0 .\end{cases}
$$

Moreover, when $-1 \leq y \leq b<1$ and $\lambda / 2+\nu+1<0$, we have

$$
F_{1}(y) \leq \text { const } m^{-2-\lambda} \text {. }
$$

Proof. The proof of the lemma requires standard calculations; a sketch of the proof is given here. We consider first the case $\nu=0$, and set $d=1-a$ and $t=m \sqrt{1-x}$. When $\lambda=-1$ we have

$$
F_{1}(y)=\frac{2}{m} \int_{0}^{m \sqrt{d}} \frac{t}{t+1} d t=O(1)
$$

while for $\lambda=-2$

$$
F_{1}(y)=2 \int_{0}^{m \sqrt{d}} \frac{t}{(t+1)^{2}} d t \leq \text { const } \log (m \sqrt{d}+1) .
$$

In the remaining cases, i.e., $\lambda \neq-1,-2$, we obtain

$$
\begin{aligned}
F_{1}(y) & \leq 2 m^{-2-\lambda} \int_{0}^{m \sqrt{d}}(t+1)^{\lambda} t d t \\
& =2 m^{-2-\lambda}\left[\frac{(m \sqrt{d}+1)^{\lambda+2}}{\lambda+2}-\frac{(m \sqrt{d}+1)^{\lambda+1}}{\lambda+1}+\frac{1}{(\lambda+1)(\lambda+2)}\right] .
\end{aligned}
$$

Thus (2.1) is proved when $\nu=0$, hence also when $\nu>0$ since in this latter case, $|x-y|^{\nu} \leq 2^{\nu}$. Next we examine the case $-1<\nu<0$. In particular, we consider first $1-m^{-2} \leq y \leq 1$ and then $-1 \leq y \leq 1-m^{-2}$.

For $1-m^{-2} \leq y \leq 1$, we split $F_{1}(y)$ as follows:

$$
F_{1}(y)=\left\{\int_{a}^{1-c m^{-2}}+\int_{1-c m^{-2}}^{1}\right\}\left(\sqrt{1-x}+m^{-1}\right)^{\lambda}|x-y|^{\nu} d x
$$


with $c>1$, and write

$$
F_{1}(y)<\int_{a}^{1-c m^{-2}}(1-x)^{\lambda / 2}(y-x)^{\nu} d x+m^{-\lambda} \int_{1-c m^{-2}}^{1}|x-y|^{\nu} d x
$$

i.e.,

$$
F_{1}(y)<\int_{a}^{1-c m^{-2}}(y-x)^{\lambda / 2+\nu} d x+m^{-\lambda} \int_{1-c m^{-2}}^{1}|x-y|^{\nu} d x .
$$

From this last bound we derive (2.1).

When $a \leq y \leq 1-m^{-2}$, we proceed differently:

$$
\begin{aligned}
F_{1}(y) & =\left\{\int_{a}^{y}+\int_{y}^{1}\right\}\left(\sqrt{1-x}+m^{-1}\right)^{\lambda}|x-y|^{\nu} d x \\
& \leq \int_{a}^{y}(1-x)^{\lambda / 2}(y-x)^{\nu} d x+\int_{y}^{1}\left(\sqrt{1-x}+m^{-1}\right)^{\lambda}(x-y)^{\nu} d x .
\end{aligned}
$$

For the first integral in the last line we obtain

$$
\begin{gathered}
\int_{a}^{y}(1-x)^{\lambda / 2}(y-x)^{\nu} d x=-\frac{1}{\nu+1} \int_{a}^{y}(1-x)^{\lambda / 2} d(y-x)^{\nu+1} \\
\leq \frac{1}{\nu+1}(y-a)^{\nu+1}(1-a)^{\lambda / 2}-\frac{\lambda}{2(\nu+1)} \int_{a}^{y}(1-x)^{\lambda / 2+\nu} d x
\end{gathered}
$$

hence upper bounds similar to (2.1).

The integral

$$
I(y)=\int_{y}^{1}\left(\sqrt{1-x}+m^{-1}\right)^{\lambda}(x-y)^{\nu} d x
$$

needs some more care. When $\lambda / 2+\nu+1 \geq 0$ we have

$$
I(y)<\int_{y}^{1}(1-x)^{\lambda / 2}(x-y)^{\nu} d x=(1-y)^{\lambda / 2+\nu+1} \int_{0}^{1} t^{\nu}(1-t)^{\lambda / 2} d t=O(1) .
$$

When $\lambda / 2+\nu+1<0$ we write

$$
\begin{aligned}
I(y)< & \int_{y}^{y+m^{-2} / 2}(1-x)^{\lambda / 2}(x-y)^{\nu} d x \\
& +\left(\frac{1}{2} m^{-2}\right)^{\nu} \int_{y+m^{-2} / 2}^{1}\left(\sqrt{1-x}+m^{-1}\right)^{\lambda} d x \\
< & \frac{1}{\nu+1} \int_{y}^{y+m^{-2} / 2}(1-x)^{\lambda / 2} d(x-y)^{\nu+1} \\
& +\frac{1}{2^{\nu}} m^{-2 \nu} \int_{a}^{1}\left(\sqrt{1-x}+m^{-1}\right)^{\lambda} d x,
\end{aligned}
$$

and easily obtain the bound $O\left(m^{-2-\lambda-2 \nu}\right)$.

The proof of (2.2) is not difficult; one needs only to consider a point $c$, $b<c<1$, and break the integral over $(-1,1)$ into the sum of two integrals:

${ }^{2}$ The case $-1 \leq y \leq a$ is simpler and we omit it. 
one over $(-1, c)$ and the other over $(c, 1)$. Since $\left(\sqrt{1-x}+m^{-1}\right)^{\lambda} \leq$ const for $-1 \leq x \leq c$ and $|x-y|^{\nu} \leq$ const for $c \leq x \leq 1$, (2.2) follows easily.

We omit the proof of the following lemma, since it is very similar to that of Lemma 1.

Lemma 2. The integral

$$
F_{2}(y)=\int_{a}^{1}\left(\sqrt{1-x}+m^{-1}\right)^{\lambda}|\log | x-y|| d x,
$$

with $-1 \leq a<1, \lambda<0$ and $|y| \leq 1$, admits the following bounds:

$$
F_{2}(y) \leq \text { const } \begin{cases}1 & \text { if } \lambda>-2, \\ \log ^{2} m & \text { if } \lambda=-2, \\ m^{-2-\lambda} \log m & \text { if } \lambda<-2 .\end{cases}
$$

Moreover, when $-1 \leq y \leq b<1$, we have

$$
F_{2}(y) \leq \text { const } \begin{cases}1 & \text { if } \lambda>-2 \\ \log m & \text { if } \lambda=-2 \\ m^{-2-\lambda} & \text { if } \lambda<-2\end{cases}
$$

Lemma 3 (see [16]). Given any function $f \in C^{r}[-1,1], r \geq 0$, there exists a polynomial $T_{m}$ of degree $m \geq 2 r+1$ such that for $x \in[-1,1]$

$$
\begin{aligned}
\left|T_{m}^{(k)}(x)-f^{(k)}(x)\right| \leq \mathrm{const}\left(\frac{\sqrt{1-x^{2}}}{m}\right)^{r-k} \omega\left(f^{(r)} ; \frac{\sqrt{1-x^{2}}}{m}\right), \\
k=0,1, \ldots, r,
\end{aligned}
$$

where $\omega(f ; \cdot)$ denotes the modulus of continuity of the function $f$ in $[-1,1]$, and

$$
\left|T_{m}^{(k)}(x)\right| \leq \operatorname{const}\left[\Delta_{m}(x)\right]^{r-k} \omega\left(f^{(r)} ; \Delta_{m}(x)\right), \quad k \geq r+1,
$$

where $\Delta_{m}(x)=\max \left\{m^{-1} \sqrt{1-x^{2}}, m^{-2}\right\}$.

Theorem 1. Let $\sigma$ and $\nu$ be real numbers, with $\sigma, \nu>-1, \sigma+\nu>-1$ and $\sigma$ not an integer. Given any positive integer $s$, there exists an algebraic polynomial $t_{m}$ of degree $m$ such that

$$
\begin{array}{r}
\left|(1-x)^{\sigma}-t_{m}(x)\right| \leq \frac{\text { const }}{m^{s+\sigma+1}}(1-x)^{\sigma / 2-(s+1) / 2}(1+x)^{\sigma / 2+(s+1) / 2} \\
-1 \leq x<1,
\end{array}
$$

moreover

$$
\begin{aligned}
& \int_{-1}^{1}\left|(1-x)^{\sigma}-t_{m}(x)\right||x-y|^{\nu} d x \\
& \quad \leq \text { const } \begin{cases}m^{-2 \sigma-2-2 \nu} & \text { if }|y| \leq 1, \nu<0, \\
m^{-2 \sigma-2} & \text { if }|y| \leq 1, \nu \geq 0, \\
\text { or }-1 \leq y \leq b<1, \nu>-1,\end{cases}
\end{aligned}
$$

where const is independent of $y$ and $m$. 
Proof. Choose an integer $s \geq 1$. Then, consider the function

$$
g(x)=(1-x)^{s+\sigma+1},
$$

which is of class $C^{s^{\prime}}[-1,1], s^{\prime}=s+[\sigma+1],{ }^{3}$ with $g^{\left(s^{\prime}\right)} \in \operatorname{Lip} \sigma^{\prime},{ }^{4} \sigma^{\prime}=\sigma+1-$ $[\sigma+1]$, and let $T_{m+s+1}$ be the polynomial defined by Lemma 3 corresponding to the function $g$. Recalling $(2.3)$, we derive $T_{m+s+1}^{(k)}(1)=0, k=0,1, \ldots, s^{\prime}$; hence, the polynomial $t_{m}(x):=(1-x)^{-s-1} T_{m+s+1}(x)$ has degree $m$. Applying (2.3) with $k=0$ and $r=s^{\prime}$, we have

$$
\begin{aligned}
& \left|(1-x)^{\sigma}-t_{m}(x)\right|=\frac{\left|g(x)-T_{m+s+1}(x)\right|}{(1-x)^{s+1}} \\
& \quad \leq \text { const }\left(\frac{\sqrt{1-x^{2}}}{m}\right)^{s^{\prime}} \frac{\omega\left(g^{\left(s^{\prime}\right)} ; \sqrt{\left(1-x^{2}\right)} / m\right)}{(1-x)^{s+1}}, \quad-1 \leq x<1 ;
\end{aligned}
$$

hence (2.5) follows.

Furthermore, from the Taylor expansion of $T_{m+s+1}$ about the point $x=1$ we obtain

$$
t_{m}(x)=\frac{T_{m+s+1}(x)}{(1-x)^{s+1}}=\frac{1}{\left(s^{\prime}+1\right) !} \frac{(1-x)^{s^{\prime}+1}}{(1-x)^{s+1}} T_{m+s+1}^{\left(s^{\prime}+1\right)}\left(\xi_{x}\right), \quad x<\xi_{x}<1 .
$$

Inserting the bound (2.4), with $r=s^{\prime}$ and $k=s^{\prime}+1$, into this last relation, we derive (2.6).

Next, consider the integral

$$
\begin{aligned}
\int_{-1}^{1} \mid & (1-x)^{\sigma}-t_{m}(x)|| x-\left.y\right|^{\nu} d x \\
\quad & \left\{\int_{-1}^{1-m^{-2}}+\int_{1-m^{-2}}^{1}\right\}\left|(1-x)^{\sigma}-t_{m}(x)\right||x-y|^{\nu} d x \\
& =: A_{1}(y)+A_{2}(y),
\end{aligned}
$$

with $|y| \leq 1$. The inequalities (2.5), with $s>\sigma+2 \nu+1$, and $1-x \geq$ $1 / 4\left(\sqrt{1-x}+m^{-1}\right)^{2},-1 \leq x \leq 1-m^{2}$, together with Lemma 1 , allow us to write

$$
\begin{aligned}
A_{1}(y) & \leq \frac{\text { const }}{m^{s+\sigma+1}} \int_{-1}^{1-m^{-2}}(1-x)^{\sigma / 2-(s+1) / 2}|x-y|^{\nu} d x \\
& \leq \frac{\text { const }}{m^{s+\sigma+1}} \int_{-1}^{1}\left(\sqrt{1-x}+m^{-1}\right)^{\sigma-(s+1)}|x-y|^{\nu} d x \\
& \leq \text { const } m^{-2 \sigma-2 \nu-2} .
\end{aligned}
$$

${ }^{3}[a]=$ integer part of $a$.

${ }^{4} \operatorname{Lip} \sigma^{\prime}$ is the classical Lipschitz space of order $\sigma^{\prime}$. 
On the other hand, for $A_{2}(y)$ we have

$$
A_{2}(y) \leq \int_{1-m^{-2}}^{1}(1-x)^{\sigma}|x-y|^{\nu} d x+\int_{1-m^{-2}}^{1}\left|t_{m}(x)\right||x-y|^{\nu} d x,
$$

hence, recalling (2.6), the upper bound

$$
\text { const } m^{-2 \sigma} \int_{1-m^{-2}}^{1}|x-y|^{\nu} d x \leq \text { const } m^{-2 \sigma-2 \nu-2} \text {, }
$$

if $\sigma \geq 0$. When $\sigma<0$, we need to examine three different cases: $1-m^{-2} \leq$ $y \leq 1,1-2 m^{-2} \leq y \leq 1-m^{-2}, y \leq 1-2 m^{-2}$, to derive for $A_{2}(y)$ the upper bound const $m^{-2 \sigma-2-2 \nu}$. Bound (2.7) for the cases $|y| \leq 1, \nu \geq 0$ and $-1 \leq y \leq b<1, \nu>-1$, is derived in a similar way.

Remark. If in (2.9) we choose $s \leq \sigma+2 \nu+1$, from Lemma 1 we obtain the bounds

$$
A_{1}(y) \leq \frac{\text { const }}{m^{s+\sigma+1}} \begin{cases}1 & \text { if } s<\sigma+2 \nu+1, \\ \log m & \text { if } s=\sigma+2 \nu+1\end{cases}
$$

for $|y| \leq 1$, which are worse than the bound of $A_{1}(y)$ obtained for $s>\sigma+$ $2 \nu+1$.

The proof of the next theorem is very similar to that of Theorem 1; we need only use Lemma 2 instead of Lemma 1.

Theorem 2. Let $\sigma>-1$ be not an integer. There exists an algebraic polynomial $t_{m}^{*}$ of degree $m$ such that

$\int_{-1}^{1}\left|(1-x)^{\sigma}-t_{m}^{*}(x)\right||\log | x-y|| d x \leq \mathrm{const}\left\{\begin{array}{l}m^{-2 \sigma-2} \log m \quad \text { if }|y| \leq 1, \\ m^{-2 \sigma-2} \text { if }-1 \leq y \leq b<1,\end{array}\right.$ where const is independent of $y$ and $m$.

The following lemma, which is a straightforward consequence of Statements $4.2,5.4$ and 5.5 in [6], is necessary to prove our subsequent results.

Lemma 4. Let $r, i, m$ be positive integers with $m \geq \max \{4(r+1), r+i\}$. Given any function $f \in C^{r}[-1,1]$, there exists a polynomial $Q_{m}$ of degree $m$ such that for $x \in[-1,1]$

$$
\begin{gathered}
Q_{m}^{(k)}( \pm 1)=f^{(k)}( \pm 1), \quad k=0,1, \ldots, r, \\
\left|Q_{m}^{(k)}(x)-f^{(k)}(x)\right| \leq \mathrm{const}\left[\frac{\sqrt{1-x^{2}}}{m}\right]^{r-k} \omega_{i}\left(f^{(r)} ; \frac{\sqrt{1-x^{2}}}{m}\right), \\
k=0,1, \ldots, r-i, \\
\left|Q_{m}^{(k)}(x)-f^{(k)}(x)\right|
\end{gathered}
$$

$$
\begin{aligned}
\leq \mathrm{const}\left[\frac{\sqrt{1-x^{2}}}{m}+\frac{1}{m^{2}}\right]^{r-k} \omega_{i}\left(f^{(r)} ; \frac{\sqrt{1-x^{2}}}{m}+\frac{1}{m^{2}}\right), \\
k=r-i+1, \ldots, r,
\end{aligned}
$$


where $\omega_{i}(f ; \delta)=\sup _{0<h \leq \delta}\left\|\Delta_{h}^{i} g\right\|_{[-1,1-i h]},{ }^{5} \delta>0$, is the ith modulus of continuity.

Theorem 3. Let $g(x)=(1-x)^{p} \log (1-x)$, with $p>0$ an integer. Given any integer $s \geq 1$, there exists an algebraic polynomial $q_{m}$ of degree $m$ such that

$$
\left|g(x)-q_{m}(x)\right| \leq \frac{\text { const }}{m^{2 p+s}}(1-x)^{-s / 2}(1+x)^{p+s / 2}, \quad-1 \leq x<1,
$$

and

$$
\left|g(x)-q_{m}(x)\right| \leq \text { const } m^{-2 p}, \quad 1-m^{-2} \leq x \leq 1 ;
$$

moreover,

$$
\begin{aligned}
& \int_{-1}^{1}\left|(1-x)^{p} \log (1-x)-q_{m}(x)\right||x-y|^{\nu} d x \\
& \leq \text { const } \begin{cases}m^{-2 p-2-2 \nu} & \text { if }|y| \leq 1,-1<\nu<0, \\
m^{-2 p-2} & \text { if }|y| \leq 1, \nu \geq 0, \\
\text { or }-1 \leq y \leq b<1, \nu>-1,\end{cases}
\end{aligned}
$$

where const is independent of $y$ and $m$.

Proof. Having fixed an integer $s \geq 1$, consider the functions

$$
\gamma(x)=(1-x)^{2 p+s} \log (1-x)
$$

and

$$
g(x)=(1-x)^{p} \log (1-x)=(1-x)^{-p-s} \gamma(x) .
$$

Notice that the function $\gamma$ is of class $C^{2 p+s-1}[-1,1]$, with $\omega_{2}\left(\gamma^{(2 p+s-1)} ; \delta\right) \leq$ const $\delta, \delta>0$.

Let $Q_{m+p+s}$ be the polynomial defined by Lemma 4 corresponding to the function $\gamma$. Recalling (2.11), we have $Q_{m+p+s}^{(k)}(1)=\gamma^{(k)}(1)=0, k=0,1, \ldots$, $2 p+s-1$; hence the polynomial $q_{m}(x)=(1-x)^{-p-s} Q_{m+p+s}(x)$ has degree $m$.

Next, define $\rho_{m}=\gamma-Q_{m+p+s}$ and use (2.12) with $r=2 p+s-1, k=0$, $i=2$. We have

$$
\begin{aligned}
& \left|g(x)-q_{m}(x)\right|=\frac{\left|\rho_{m}(x)\right|}{(1-x)^{p+s}} \\
& \leq \text { const }\left[\frac{\sqrt{1-x^{2}}}{m}\right]^{2 p+s-1} \omega_{2}\left(\gamma^{(2 p+s-1)} ; \frac{\sqrt{1-x^{2}}}{m}\right)(1-x)^{-p-s}, \\
& -1 \leq x<1 ;
\end{aligned}
$$

hence (2.14) follows.

Furthermore, from the Taylor expansion of $\rho_{m}$ about the point $x=1$ we derive

$$
\rho_{m}(x)=\frac{(x-1)^{2 p+s-1}}{(2 p+s-1) !} \rho_{m}^{(2 p+s-1)}\left(\xi_{x}\right), \quad x<\xi_{x}<1 .
$$

\footnotetext{
${ }^{5}$ By $\Delta_{h}^{i} g=\Delta_{h}^{i} g(x)$ we denote the $i$ th finite difference with step $h$ at the point $x$.
} 
Recalling (2.13), with $k=r=2 p+s-1$ and $i=2$, from this last relation we obtain

$$
\begin{aligned}
&\left|g(x)-q_{m}(x)\right| \leq \frac{(1-x)^{p-1}}{(2 p+s-1) !}\left|\rho_{m}^{(2 p+s-1)}\left(\xi_{x}\right)\right| \\
& \leq \operatorname{const}(1-x)^{p-1} \omega_{2}\left(\gamma^{(2 p+s-1)} ; \frac{\sqrt{1-\xi_{x}^{2}}}{m}+\frac{1}{m^{2}}\right), \\
& 1-m^{-2} \leq x \leq 1 .
\end{aligned}
$$

From this last bound we deduce (2.15).

Now we are ready to examine the integral

$$
\begin{aligned}
\int_{-1}^{1} \mid & g(x)-q_{m}(x)|| x-\left.y\right|^{\nu} d x \\
& =\left\{\int_{-1}^{1-m^{-2}}+\int_{1-m^{-2}}^{1}\right\}\left|g(x)-q_{m}(x)\right||x-y|^{\nu} d x \\
= & : B_{1}(y)+B_{2}(y) .
\end{aligned}
$$

To bound $B_{1}(y)$, we use $(2.14)$ :

$$
\begin{aligned}
B_{1}(y) & \leq \frac{\text { const }}{m^{2 p+s}} \int_{-1}^{1-m^{-2}}(1-x)^{-s / 2}|x-y|^{\nu} d x \\
& \leq \frac{\text { const }}{m^{2 p+s}} \int_{-1}^{1}\left(\sqrt{1-x}+m^{-1}\right)^{-s}|x-y|^{\nu} d x .
\end{aligned}
$$

Recalling (2.15), for $B_{2}(y)$ we have

$$
B_{2}(y) \leq \frac{\text { const }}{m^{2 p}} \int_{1-m^{-2}}^{1}|x-y|^{\nu} d x .
$$

Finally, inserting (2.18) and (2.19) in (2.17) and applying Lemma 1, we deduce (2.16).

In a very similar way, with Lemma 1 replaced by Lemma 2, we can prove the following result.

Theorem 4. Let $p>0$ be an integer. There exists an algebraic polynomial $q_{m}^{*}$ of degree $m$ such that

$$
\begin{aligned}
& \int_{-1}^{1}\left|(1-x)^{p} \log (1-x)-q_{m}^{*}(x)\right||\log | x-y|| d x \\
& \quad \leq \text { const } \begin{cases}m^{-2 p-2} \log m & \text { if }|y| \leq 1 \\
m^{-2 p-2} & \text { if }-1 \leq y \leq b<1,\end{cases}
\end{aligned}
$$

where const is independent of $y$ and $m$.

Remark. To our knowledge, the existence of polynomials $t_{m}, t_{m}^{*}, q_{m}$ and $q_{m}^{*}$ which satisfy the bounds given in Theorems 1-4 is new. Furthermore, when 
in Theorems 1 and 3 above we set $\nu=0$ we obtain that the $L_{1}$-errors of $t_{m}$ and $q_{m}$ are of the same order as the $L_{1}$-errors associated with the corresponding best $L_{1}$-approximation polynomial (see [1,3]). Notice, however, that our polynomials $t_{m}$ and $q_{m}$, which in general are not the best $L_{1}$-approximations, satisfy the extra properties $(2.5),(2.6),(2.14)$ and $(2.15)$, which are essential in our proofs of the following Theorems 5-8.

We are now ready to prove our main convergence results.

\section{CONVERgence estimates for PRODUCt RUles (1.3)}

Let $w$ be a generalized smooth Jacobi weight $(w \in \mathrm{GJB})$, that is,

$$
w(x)=\varphi(x)(1-x)^{\alpha}(1+x)^{\beta}, \quad-1 \leq x \leq 1,
$$

where $\alpha, \beta>-1, \varphi>0$ is continuous and the modulus of continuity $\omega$ of $\varphi$ satisfies $\int_{0}^{1} \omega(\varphi ; \delta) \delta^{-1} d \delta<\infty$. Let $\left\{x_{m, k}(w), k=1, \ldots, m\right\}$ denote the $m$ zeros of the $m$ th-degree generalized Jacobi orthogonal polynomial

$$
p_{m}(w ; x)=\alpha_{m}(w) x^{m}+\text { lower-degree terms }, \quad \alpha_{m}(w)>0,
$$

and let $\lambda_{m, k}(w), i=1, \ldots, m$, be the corresponding Christoffel constants. For a given weight $w \in$ GJB and a bounded function $f$, the corresponding Lagrange interpolating polynomial is denoted by $L_{m}(w ; f)$ and is given by the expression

$$
L_{m}(w ; f ; x)=\sum_{k=1}^{m} f\left(x_{m, k}\right) l_{m, k}(w ; x),
$$

where for the fundamental polynomials $l_{m, k}(w)$ we have (see [12])

$$
l_{m, k}(w ; x)=\frac{\alpha_{m-1}(w)}{\alpha_{m}(w)} \lambda_{m, k}(w) p_{m-1}\left(w ; x_{m, k}(w)\right) \frac{p_{m}(w ; x)}{x-x_{m, k}(w)}
$$

Furthermore, we denote by $L_{m}^{(i, j)}(w ; f ; x), i, j \in\{0,1\}$, the interpolating polynomial of degree at most $m+i+j-1$ which satisfies

$$
L_{m}^{(i, j)}\left(w ; f ; x_{m, k}(w)\right)=f\left(x_{m, k}(w)\right), \quad 1-i \leq k \leq m+j,
$$

where $x_{m, 0}(w)=-1, x_{m, m+1}(w)=1$.

The polynomial $L_{m}^{(i, j)}(w ; f)$ can be explicitly represented in the form

$$
\begin{aligned}
L_{m}^{(i, j)}(w ; f ; x)= & \sum_{k=1}^{m} f\left(x_{m, k}(w)\right) h_{m, k}(w ; x)+\delta_{1, j} f(-1) h_{m, 0}(w ; x) \\
& +\delta_{1, i} f(1) h_{m, m+1}(w ; x),
\end{aligned}
$$

where

$$
\begin{aligned}
& h_{m, k}(w ; x) \\
& 4)=(1-x)^{i}(1+x)^{j}\left(1-x_{m, k}(w)\right)^{-i}\left(1+x_{m, k}(w)\right)^{-j} l_{m, k}(w ; x) \\
& \\
& \text { for } 1 \leq k \leq m,
\end{aligned}
$$




$$
\begin{aligned}
& h_{m, 0}(w ; x)=\frac{1-x}{2 p_{m}(w ;-1)} p_{m}(w ; x), \\
& h_{m, m+1}(w ; x)=\frac{1+x}{2 p_{m}(w ; 1)} p_{m}(w ; x),
\end{aligned}
$$

and $\delta_{r, s}$ denotes the Kronecker symbol. The quadrature sum of type (1.3) can now be defined as follows:

$$
\begin{aligned}
I_{m}(f ; y):= & \int_{-1}^{1} K(x, y) L_{m}^{(i, j)}(w ; f ; x) d x \\
= & \sum_{k=1}^{m} w_{m, k}(y) f\left(x_{m, k}(w)\right)+\delta_{1, j} w_{m, 0}(y) f(-1) \\
& +\delta_{1, i} w_{m, m+1}(y) f(1)
\end{aligned}
$$

with

$$
w_{m, k}(y)=\int_{-1}^{1} K(x, y) h_{m, k}(w ; x) d x, \quad 1-i \leq k \leq m+j .
$$

Practical algorithms to construct the weights $\left\{w_{m, k}(y)\right\}$ efficiently are presented in [11].

Consider the error term of (1.3), i.e.,

$$
R_{m}(f ; y)=\int_{-1}^{1} K(x, y) f(x) d x-I_{m}(f ; y) .
$$

Since the weights $\left\{w_{m, k}(y)\right\}$ satisfy (see $\left.[19,20]\right)$ the relation

$$
\lim _{m \rightarrow \infty} \sum_{k=0}^{m+1}\left|w_{m, k}(y)\right|=\int_{-1}^{1}|K(x, y)| d x<\infty,
$$

which, recalling [13], holds even uniformly with respect to $y$, we have that $R_{m}(f)=O\left(m^{-n}\right)$ whenever $f \in C^{n}[-1,1]$. Thus, in the following we may assume, without any loss of generality, $f(x)=(1-x)^{\sigma}, \sigma>-1$, and examine the behavior of $R_{m}(f)$.

In (3.5) we have $R_{m}(f)=0$ whenever $f$ is a polynomial of degree $m-1$; hence, given any polynomial $P_{m-1}$ of degree $m-1$, we can write

$$
\begin{aligned}
R_{m}(f ; y)= & \int_{-1}^{1} K(x, y)\left[f(x)-P_{m-1}(x)\right] d x \\
& -\int_{-1}^{1} K(x, y)\left[L_{m}^{(i, j)}\left(f-P_{m-1} ; x\right)\right] d x
\end{aligned}
$$

By a proper choice of the sequence of polynomials $\left\{P_{m-1}\right\}$, we will be able, at least in the two common cases $K(x, y)=|x-y|^{\nu}$ and $K(x, y)=\log |x-y|$, to derive upper bounds for the two terms

$$
R_{m}^{(1)}(f ; y)=\int_{-1}^{1}|K(x, y)|\left|f(x)-P_{m-1}(x)\right| d x
$$




$$
R_{m}^{(2)}(f ; y)=\int_{-1}^{1}|K(x, y)|\left|L_{m}^{(i, j)}\left(f-P_{m-1} ; x\right)\right| d x
$$

Before proceeding any further, we need another preliminary result.

For any $x \in(-1,1), m \in \mathbb{N}$, we denote by $x_{c(m)}(w)=x_{m, c}(w)$ the knot closest to $x$, defined by

$$
x_{m, c}(w)= \begin{cases}x_{m, d}(w) & \text { if } x-x_{m, d}(w) \leq x_{m, d+1}(w)-x, \\ x_{m, d+1}(w) & \text { if } x-x_{m, d}(w)>x_{m, d+1}(w)-x,\end{cases}
$$

where $x_{m, d}(w) \leq x \leq x_{m, d+1}(w)$ for some $d \in\{0,1, \ldots, m\}$. Then we introduce the function

$$
S_{m}^{\rho}(w ; x)=\sum_{\substack{k=1 \\ k \neq c}}^{m} \frac{\left(1-x_{m, k}(w)\right)^{\rho}}{m\left|x-x_{m, k}(w)\right|}
$$

and recall (see [5]) the following lemma.

Lemma 5. If the weight function $w \in \mathrm{GJB}$ is defined by (3.1), then for every $x \in[-1,1]$

$$
S_{m}^{\rho}(w ; x) \leq \mathrm{const}\left\{\begin{array}{l}
m^{-2 \rho-1}\left(\sqrt{1-x}+m^{-1}\right)^{-2} \\
+\left(\sqrt{1-x}+m^{-1}\right)^{2 \rho-1} \log m \quad \text { if } \rho<-1 / 2, \\
\left(\sqrt{1-x}+m^{-1}\right)^{2 \rho-1} \log m \quad \text { if }-1 / 2 \leq \rho \leq 1 / 2, \\
\left(\sqrt{1-x}+m^{-1}\right)^{2 \rho-1} \log m+1 \quad \text { if } \rho>1 / 2 .
\end{array}\right.
$$

Theorem 5. Let $f(x)=(1-x)^{\sigma}, \sigma>-1$ (not an integer) and $\nu>-1$, with $\sigma+\nu>-1$, and assume $i, j \in\{0,1\}$ in (3.3). When the indices $\alpha, \beta$ of the weight $w \in \mathrm{GJB}$ satisfy the condition

$$
\alpha \geq-1 / 2+2 i, \quad-1<\beta \leq 2 j+3 / 2
$$

and $\sigma>0$ if $i=1$, we have

$$
\begin{aligned}
& \int_{-1}^{1}\left|f(x)-L_{m}^{(i, j)}(w ; f ; x)\right||x-y|^{\nu} d x \\
& \quad \leq \operatorname{const}\left\{\begin{array}{rr}
m^{-2-2 \sigma-2 \nu} \log m & \text { if }|y| \leq 1, \nu<0 \\
m^{-2-2 \sigma} \log m & \text { if }|y| \leq 1, \nu \geq 0 \\
\text { or }-1 \leq y \leq b<1, \nu>-1,
\end{array}\right.
\end{aligned}
$$

where const is independent of $y$ and $m$. 
Proof. Let $t_{m-1}$ be the polynomial of Theorem 1 and consider the inequality

$$
\begin{aligned}
\int_{-1}^{1}\left|f(x)-L_{m}^{(i, j)}(w ; f ; x)\right||x-y|^{\nu} d x \\
\leq \int_{-1}^{1}\left|f(x)-t_{m-1}(x)\right||x-y|^{\nu} d x \\
\quad+\int_{-1}^{1}\left|L_{m}^{(i, j)}\left(w ; f-t_{m-1} ; x\right)\right||x-y|^{\nu} d x \\
=: I_{1}(y)+I_{2}(y) .
\end{aligned}
$$

The integral $I_{1}(y)$ can be bounded using (2.7), while for $I_{2}(y)$ we need some more calculation. First we set $r_{m-1}=f-t_{m-1}$ and recall that $r_{m-1}(1)=0$, when $\sigma>0$, and $r_{m-1}(-1)=0$; then, since we can take $i=1$ only if $\sigma>0$, we write

$$
\begin{aligned}
\left|L_{m}^{(i, j)}\left(w ; r_{m-1} ; x\right)\right| \leq & \left|h_{m, c}(w ; x)\right|\left|r_{m-1}\left(x_{m, c}(w)\right)\right| \\
& +\sum_{\substack{k=1 \\
k \neq c}}^{m}\left|h_{m, k}(w ; x)\right|\left|r_{m-1}\left(x_{m, k}(w)\right)\right|,
\end{aligned}
$$

where $c$ is the index corresponding to the closest knot to $x$.

Notice that (see [12, proof of Theorem 33, p. 171])

$$
\left|l_{m, c}(w ; x)\right| \leq \text { const }, \quad-1 \leq x \leq 1 ;
$$

furthermore, relation (3.2), together with inequalities (1), (14) and (20) in [14, pp. 671, 673,674], gives us

$$
\begin{aligned}
& \left|l_{m, k}(w ; x)\right| \\
& \quad \leq \operatorname{const} \frac{\left(1-x_{m, k}(w)\right)^{\alpha / 2+3 / 4}\left(1+x_{m, k}(w)\right)^{\beta / 2+3 / 4}\left|p_{m}(w ; x)\right|}{m\left|x-x_{m, k}(w)\right|}, \quad k \neq c .
\end{aligned}
$$

Thus, taking into account (3.4), by (2.5) we deduce

$$
\begin{aligned}
& \left|L_{m}^{(i, j)}\left(w ; r_{m-1} ; x\right)\right| \\
& \leq \mathrm{const}\left\{\frac{\left(1-x_{\left.\left.m, c^{(w)}\right)\right)^{(\sigma-s) / 2}}\right.}{m^{\sigma+s}}+\frac{(1-x)^{i}(1+x)^{j}\left|p_{m}(w ; x)\right|}{m^{\sigma+s}}\right. \\
& \left.\cdot \sum_{\substack{k=1 \\
k \neq c}}^{m} \frac{\left(1-x_{m, k}(w)\right)^{(\alpha+\sigma) / 2+3 / 4-s / 2-i}\left(1+x_{m, k}(w)\right)^{(\beta+\sigma) / 2+3 / 4+s / 2-j}}{m\left|x-x_{m, k}(w)\right|}\right\}
\end{aligned}
$$

for any integer $s \geq 1$. Since

$$
1-x_{m, c}(w) \geq \text { const }\left(\sqrt{1-x}+m^{-1}\right)^{2}
$$

and

$$
\left|p_{m}(w ; x)\right| \leq \text { const }\left(\sqrt{1-x}+m^{-1}\right)^{-\alpha-1 / 2}\left(\sqrt{1+x}+m^{-1}\right)^{-\beta-1 / 2}
$$


(see [14, inequalities (11), (15), p. 673]), inequality (3.8) can be rewritten as follows:

$$
\begin{aligned}
\left|L_{m}^{(i, j)}\left(w ; r_{m-1} ; x\right)\right| & \\
\leq \frac{\text { const }}{m^{s+\sigma}} & \left\{\left(\sqrt{1-x}+m^{-1}\right)^{\sigma-s}+\left(\sqrt{1-x}+m^{-1}\right)^{2 i-\alpha-1 / 2}\right. \\
& \left.\cdot\left(\sqrt{1+x}+m^{-1}\right)^{\eta} \sum_{\substack{k=1 \\
k \neq c}}^{m} \frac{\left(1-x_{m, k}(w)\right)^{(\alpha+\sigma) / 2+3 / 4-s / 2-i}}{m\left|x-x_{m, k}(w)\right|}\right\},
\end{aligned}
$$

where $\eta=2 j-\beta-1 / 2$.

Assuming that condition (3.7) is satisfied and $i=0$, we apply the first inequality in (3.6) for $s>\alpha+\sigma+5 / 2$; we obtain

$$
\begin{aligned}
& \left|L_{m}^{(0, j)}\left(w ; r_{m-1} ; x\right)\right| \\
& \leq \operatorname{const} \frac{\left(\sqrt{1+x}+m^{-1}\right)^{\eta}}{m^{s+\sigma}} \\
& \quad \cdot\left[\frac{\left(\sqrt{1-x}+m^{-1}\right)^{-\alpha-5 / 2}}{m^{(\alpha+\sigma+5 / 2-s}}+\left(\sqrt{1-x}+m^{-1}\right)^{\sigma-s} \log m\right]
\end{aligned}
$$

hence

$$
\begin{array}{r}
I_{2}(y) \leq \frac{\text { const }}{m^{s+\sigma}}\left\{\left[\frac{1}{m^{\alpha+\sigma+5 / 2-s}}+\log m\right] \int_{-1}^{0}\left(\sqrt{1+x}+m^{-1}\right)^{\eta}|x-y|^{\nu} d x\right. \\
+\frac{1}{m^{\alpha+\sigma+5 / 2-s}} \int_{0}^{1}\left(\sqrt{1-x}+m^{-1}\right)^{-\alpha-5 / 2}|x-y|^{\nu} d x \\
\left.\quad+\log m \int_{0}^{1}\left(\sqrt{1-x}+m^{-1}\right)^{\sigma-s}|x-y|^{\nu} d x\right\} .
\end{array}
$$

Finally, recalling that $\alpha \geq-1 / 2$ and that the condition $\beta \leq 2 j+3 / 2$ implies $\eta \geq-2$, the assertion follows by Lemma 1 . 
On the other hand, if $i=1 \quad(\sigma>0)$, then (3.9) becomes

$$
\begin{aligned}
& \left|L_{m}^{(1, j)}\left(w ; r_{m-1} ; x\right)\right| \leq \frac{\text { const }}{m^{s+\sigma}}\left\{\left(\sqrt{1-x}+m^{-1}\right)^{\sigma-s}\right. \\
& +\left(\sqrt{1-x}+m^{-1}\right)^{3 / 2-\alpha}\left(\sqrt{1+x}+m^{-1}\right)^{\eta} \\
& \left.\cdot \sum_{\substack{k=1 \\
k \neq c}}^{m} \frac{\left(1-x_{m, k}(w)\right)^{(\alpha+\sigma) / 2-1 / 4-s / 2}}{m\left|x-x_{m, k}(w)\right|}\right\}
\end{aligned}
$$

that is, using Lemma 5,

$$
\begin{aligned}
& \left|L_{m}^{(1, j)}\left(w ; r_{m-1} ; x\right)\right| \\
& \leq \operatorname{const} \frac{\left(\sqrt{1-x}+m^{-1}\right)^{\eta}}{m^{s+\sigma}}\left[\frac{\left(\sqrt{1-x}+m^{-1}\right)^{-\alpha-1 / 2}}{m^{\alpha+\sigma+1 / 2-s}}\right. \\
& \left.+\left(\sqrt{1-x}+m^{-1}\right)^{\sigma-s} \log m\right] .
\end{aligned}
$$

Also in this case, the proof of the theorem follows by Lemma 1 and the assumptions on $\alpha$ and $j$.

Theorem 6. Let $g(x)=(1-x)^{p} \log (1-x)$ with $p>0$ an integer, and assume $i, j \in\{0,1\}$ in (3.3). When the indices $\alpha, \beta$ of the weight $w \in$ GJB satisfy the condition

$$
\alpha \geq-1 / 2+2 i, \quad-1<\beta \leq 2 j+3 / 2,
$$

we have

$$
\begin{aligned}
& \int_{-1}^{1}\left|g(x)-L_{m}^{(i, j)}(w ; g ; x)\right||x-y|^{\nu} d x \\
& \leq \text { const }\left\{\begin{aligned}
& m^{-2 p-2-2 \nu} \log m \text { if }|y| \leq 1,-1<\nu<0, \\
& m^{-2 p-2} \log m \text { if }|y| \leq 1, \nu \geq 0, \\
& \text { or }-1 \leq y \leq b<1, \nu>-1,
\end{aligned}\right.
\end{aligned}
$$

where const is independent of $y$ and $m$.

Proof. Choosing the polynomial $q_{m-1}$ of Theorem 3, and proceeding as in the proof of the previous theorem, we derive (3.10).

We also omit the proofs of the following theorems, since they are very similar to those of Theorems 5 and 6 , making use of the polynomials $t_{m-1}^{*}$ and $q_{m-1}^{*}$ of Theorems 2 and 4. 
Theorem 7. Let $f(x)=(1-x)^{\sigma}, \sigma>-1$ (not an integer) and assume $i, j \in$ $\{0,1\}$ in (3.3). When the indices $\alpha, \beta$ of the weight $w \in \mathrm{GJB}$ satisfy the condition

$$
\alpha \geq-1 / 2+2 i, \quad-1<\beta \leq 2 j+3 / 2,
$$

and $\sigma>0$ if $i=1$, we have

$$
\begin{aligned}
& \int_{-1}^{1}\left|f(x)-L_{m}^{(i, j)}(w ; f ; x)\right||\log | x-y|| d x \\
& \quad \leq \operatorname{const} \begin{cases}m^{-2 \sigma-2} \log ^{2} m & \text { if }|y| \leq 1, \\
m^{-2 \sigma-2} \log m & \text { if }-1 \leq y \leq b<1,\end{cases}
\end{aligned}
$$

where const is independent of $y$ and $m$.

Theorem 8. Let $g(x)=(1-x)^{p} \log (1-x)$ with $p>0$ an integer, and assume $i, j \in\{0,1\}$ in (3.3). When the indices $\alpha, \beta$ of the weight $w \in \mathrm{GJB}$ satisfy the condition

$$
\alpha \geq-1 / 2+2 i, \quad-1<\beta \leq 2 j+3 / 2,
$$

we have

$$
\begin{aligned}
& \int_{-1}^{1}\left|g(x)-L_{m}^{(i, j)}(w ; g ; x)\right||\log | x-y|| d x \\
& \quad \leq \text { const } \begin{cases}m^{-2 p-2} \log ^{2} m & \text { if }|y| \leq 1, \\
m^{-2 p-2} \log m & \text { if }-1 \leq y \leq b<1,\end{cases}
\end{aligned}
$$

where const is independent of $y$ and $m$.

Finally, we observe that all previous results remain true with the term $(1+x)^{\sigma}$ instead of $(1-x)^{\sigma}$, or with the term $\left(1-x^{2}\right)^{\sigma}$; however, in these cases the condition $-1 \leq y \leq b<1$ should be replaced by $-1<b \leq y \leq 1$ and $|y| \leq b<1$, respectively.

Remark. In (1.3), we could also assume $f(x)=(1-x)^{\rho}(1+x)^{\tau} f_{0}(x), f_{0}(x)=$ $(1-x)^{\sigma}$, and consider product rules of type

$$
\int_{-1}^{1}(1-x)^{\rho}(1+x)^{\tau} K(x, y) f_{0}(x) d x \approx \sum_{i=1}^{m} w_{m, i}(y) f_{0}\left(x_{m, i}\right),
$$

i.e., introduce the weight function $(1-x)^{\rho}(1+x)^{\tau}$ in the integrals of Theorems 1 through 8 . The modifications of all our results are straightforward. We find that our bounds are not affected by the introduction of the weight function above. For example, in Theorems 1 and 5 we have

$$
\begin{aligned}
& \int_{-1}^{1}(1-x)^{\rho}(1+x)^{\tau}\left|(1-x)^{\sigma}-t_{m}(x)\right||x-y|^{\prime \prime} d x \\
& \quad \leq \text { const }\left\{\begin{array}{lr}
m^{-2 \rho-2 \sigma-2-2 \nu} & \text { if }|y| \leq 1, \nu<0, \\
m^{-2 \rho-2 \sigma-2} & \text { if }|y| \leq 1, \nu \geq 0 \\
\text { or }-1 \leq y \leq b<1, \nu>-1,
\end{array}\right.
\end{aligned}
$$


and

$$
\begin{aligned}
& \int_{-1}^{1}(1-x)^{\rho}(1+x)^{\tau}\left|f_{0}(x)-L_{m}^{(i, j)}\left(w ; f_{0} ; x\right)\right||x-y|^{\nu} d x \\
& \leq \text { const }\left\{\begin{array}{cc}
m^{-2-2 \sigma-2 \nu}\left(m^{-2 \tau}+m^{-2 \rho} \log m\right) & \text { if }|y| \leq 1, \nu<0, \\
m^{-2-2 \sigma}\left(m^{-2 \tau}+m^{-2 \rho} \log m\right) & \text { if }|y| \leq 1, \nu \geq 0 \\
\text { or }-1 \leq y \leq b<1, \\
\nu>-1,
\end{array}\right.
\end{aligned}
$$

respectively. This means that as long as $\sigma$ is not an integer, ${ }^{6}$ i.e., our function $f(x)$ in $(1.3)$ is indeed of the form $(1-x)^{\rho}(1+x)^{\tau} f_{0}(x)$ with $\rho, \tau$ known and $f_{0}(x)$ sufficiently smooth in $[-1,1]$, there are no improvements in our bounds by interpolating $f_{0}(x)$ instead of $f(x)$.

\section{ACKNOWLEDGMENT}

The authors thank the referee for his helpful comments.

\section{BIBLIOGRAPHY}

1. N. I. Achieser, Theory of approximation, Ungar, New York, 1956.

2. K. E. Atkinson, A survey of numerical methods for the solution of Fredholm integral equations of the second kind, SIAM, Philadelphia, 1976.

3. H. Brass, A remark on best $L^{1}$-approximation by polynomials, J. Approx. Theory 52 (1988), 359-361.

4. M. M. Chawla and M. K. Jain, Error estimates for the Gauss quadrature formula, Math. Comp. 22 (1980), 91-97.

5. G. Criscuolo and G. Mastroianni, Mean and uniform convergence of quadrature rules for evaluating the finite Hilbert transform, J. Approx. Theory (to appear).

6. H. Gonska and E. Hinnemann, Punktweise Abschätzungen zur Approximation durch algebraische Polynome, Acta Math. Hungar. 46 (1985), 243-254.

7. I. G. Graham, Singularity expansions for the solutions of second kind Fredholm integral equations with weakly singular convolution kernels, J. Integral Equations 4 (1982), 1-30.

8. M. Küntz, Asymptotic error bounds for a class of interpolatory quadratures, SIAM J. Numer. Anal. 21 (1984), 167-175.

9. D. S. Lubinsky and P. Rabinowitz, Rates of convergence of Gaussian quadrature for singular integrands, Math. Comp. 43 (1984), 219-242.

10. G. Monegato and V. Colombo, Product integration for the linear transport equation in slab geometry, Numer. Math. 52 (1988), 219-240.

11. G. Monegato, Orthogonal polynomials and product integration for one-dimensional Fredholm integral equations with "nasty" kernels, Problems and Methods in Mathematical Physics (F. Kuhnert and B. Silbermann, eds.), Teubner-Texte zur Mathematik, 9. TMP, Leipzig, 1988, pp. 185-192.

12. P. Nevai, Orthogonal polynomials, Mem. Amer. Math. Soc. No. 213 (1979).

13. __ Mean convergence of Lagrange interpolation. I, J. Approx. Theory 18 (1976), 363-377.

14. 669-698.

\footnotetext{
${ }^{6}$ In this case, the remainder would be zero for $m$ sufficiently large.
} 
15. G. R. Richter, On weakly singular Fredholm integral equations with displacement kernels, J. Math. Anal. Appl. 55 (1976), 32-42.

16. P. O. Runck, Bemerkungen zu den Approximationssätzen von Jackson und Jackson-Timan, Abstrakte Räume und Approximation (P. L. Butzer and B. Szökefalvi-Nagy, eds.), ISNM 10, Birkhäuser Verlag, Basel, 1969, pp. 303-308.

17. C. Schneider, Regularity of the solution to a class of weakly singular Fredholm integral equations of the second kind, Integral Equations Operator Theory 2 (1979), 62-68.

18. $\ldots$, Product integration for weakly singular integral equations, Math. Comp. 36 (1981), 207-213.

19. I. H. Sloan and W. E. Smith, Properties of interpolatory product integration rules, SIAM J. Numer. Anal. 19 (1982), 427-442.

20. W. E. Smith and I. H. Sloan, Product-integration rules based on the zeros of Jacobi polynomials, SIAM J. Numer. Anal. 17 (1980), 1-13.

21. G. Vainikko and A. Pedas, The properties of solutions of weakly singular integral equations, J. Austral. Math. Soc. Ser. B 22 (1981), 419-430.

22. G. Vainikko and P. Uba, A piecewise polynomial approximation to the solution of an integral equation with weakly singular kernel, J. Austral. Math. Soc. Ser. B 22 (1981), 431-438.

Istituto per Applicazioni della Matematica, C.N.R., I-80131 Napoli, Italy

Istituto di Matematica, Università della Basilicata, I-85100 Potenza, Italy

Dipartimento di Matematica, Politecnico di Torino, I-10129 Torino, Italy 